\title{
Supplementary Material: Bayesian Tensor Response Regression With an Application to Brain Activation Studies
}

\author{
Rajarshi Guhaniyogi ${ }^{1}$ and Daniel Spencer ${ }^{2}$ \\ ${ }^{1}$ Department of Statistics, Baskin School of Engineering, 1156 High Street Santa Cruz, CA \\ 95060 \\ e-mail: rguhaniy@ucsc.edu \\ ${ }^{2}$ Department of Statistics, School of Arts and Sciences, 901 E 10th St Informatics West, \\ Bloomington, IN 47408 \\ e-mail: danspen@iu.edu
}

\begin{abstract}
The supplementary material contains posterior consistency in tensor response regression, proofs of the posterior consistency results, posterior sampling algorithm and a picture of the Montreal Neurological Institute $(\mathrm{MNI})$ atlas.
\end{abstract}

\section{Posterior consistency in tensor response regression}

\subsection{Notations}

In what follows, we add a subscript $(T)$ to the dimensions of tensor margins $p_{1,(T)}, \ldots, p_{D,(T)}$ and the number of predictors $m_{(T)}$ to indicate that the size of both the response tensor $\boldsymbol{Y}_{t}$ and covariates $\boldsymbol{x}_{t}$ can increase with the sample size $T$. This asymptotic paradigm is also meant to capture the fact that the number of cells $\prod_{j=1}^{D} p_{j,(T)}$ is typically larger than the sample size $T$ for each tensor coefficient $\boldsymbol{\Gamma}_{1,(T)}, . ., \boldsymbol{\Gamma}_{m_{(T)},(T)}$. Define $\boldsymbol{\Gamma}$ as a $\Re^{m} \otimes_{j=1}^{D} \Re^{p_{j}}$ tensor with the $\left(v_{1}, . ., v_{D}, k\right)$ th cell given by the $\left(v_{1}, \ldots, v_{D}\right)$ th cell of $\boldsymbol{\Gamma}_{k,(T)}$. Naturally, the tensor coefficient $\boldsymbol{\Gamma}$ and tensor margins $\boldsymbol{\gamma}_{j, k}^{(r)}$ s are also functions of the sample size $T$ and we denote them by $\boldsymbol{\Gamma}_{(T)}$ and $\boldsymbol{\gamma}_{j, k,(T)}^{(r)}$ s, respectively. We use superscript (0) to indicate true parameters, e.g. the true tensor regression parameter and the true error variance are denoted by $\boldsymbol{\Gamma}_{(T)}^{(0)}$ and $\sigma^{(0) 2}$, respectively. For simplicity, we assume that $\sigma^{2}=\sigma^{(0) 2}$ is known and fixed at 1 . We also assume that $\kappa$ is fixed and known, so that $\operatorname{var}\left(\boldsymbol{E}_{\boldsymbol{v}}\right)=\boldsymbol{R}$ is fixed, where $\boldsymbol{E}_{\boldsymbol{v}}=\left(E_{1, \boldsymbol{v}}, \ldots, E_{T, \boldsymbol{v}}\right)^{\prime}$. While $\kappa$ and $\sigma^{2}$ are unknown in practice and are assigned prior distributions, our setup assumes them to be fixed and known. Indeed, assuming parameters $\sigma^{2}$ and $\kappa$ to be known for the theoretical study is somewhat restrictive, but not very impractical as it is known that the theoretical results obtained by assuming these parameters as known constants are equivalent to those obtained by assigning priors with bounded supports on these parameters (Van der Vaart and Van Zanten, 2009). The parameter $\kappa$ belongs to $(-1,1)$ and is assigned a 
uniform prior with bounded support. Although we assign inverse-gamma prior with an unbounded prior support for $\sigma^{2}$, truncating the prior distribution at a large value would not have perhaps altered the results much. Thus, this is not a strict restriction, but a mild assumption to simplify calculation. Moreover, assuming parameters within the variance structure fixed and known is a common practice in asymptotic studies, see Van der Vaart and Van Zanten (2011). For vectors, we let $\|\cdot\|_{2}$ denote the $L_{2}$-norm, $\|\cdot\|_{1}$ denote the $L_{1}$-norm and $\|\cdot\|_{\infty}$ denote the $L_{\infty}$ norm. With a slight abuse of notation, for a $D$-dimensional tensor object $\boldsymbol{A}$, the $L_{1}, L_{2}$ and $L_{\infty}$ norms are defined as $\|\boldsymbol{A}\|_{1}=\sum_{v_{1}, \ldots, v_{D}}\left|A_{v_{1}, \ldots, v_{D}}\right|$, $\|\boldsymbol{A}\|_{2}=\sqrt{\sum_{v_{1}, \ldots, v_{D}} A_{v_{1}, \ldots, v_{D}}^{2}}$ and $\|\boldsymbol{A}\|_{\infty}=\max _{v_{1}, \ldots, v_{D}}\left|A_{v_{1}, \ldots, v_{D}}\right| \cdot\|\cdot\|_{0}$ denotes the $L_{0}$-norm, i.e., the number of non-zero entries, for both vectors and tensors. Further, assume $\mathcal{F}_{1}=\left\{\boldsymbol{h}_{1}=\left(v_{1}, \ldots, v_{D}\right): 1 \leq v_{1} \leq p_{1,(T)}, \ldots, 1 \leq v_{D} \leq p_{D,(T)}\right\}$, $\mathcal{F}_{2}=\left\{h_{2}=v_{D+1}: 1 \leq v_{D+1} \leq m_{(T)}\right\}$. Denote $\zeta^{(0)}=\left\{\left(\boldsymbol{h}_{1}, h_{2}\right): \Gamma_{\boldsymbol{h}_{1}, h_{2},(T)}^{(0)} \neq\right.$ $\left.0, \boldsymbol{h}_{1} \in \mathcal{F}_{1}, h_{2} \in \mathcal{F}_{2}\right\}$ as a set of indices corresponding to the nonzero cells of the true tensor coefficient, and also denote $\zeta_{1}^{(0)}=\left\{\boldsymbol{h}_{1} \in \mathcal{F}_{1}: \Gamma_{\boldsymbol{h}_{1}, h_{2},(T)}^{(0)} \neq\right.$ 0 , for some $\left.h_{2} \in \mathcal{F}_{2}\right\}$. Similarly, for any set $\zeta \subseteq \mathcal{F}_{1} \times \mathcal{F}_{2}$, define $\zeta_{1}=\left\{\boldsymbol{h}_{1} \in \mathcal{F}_{1}\right.$ : $\left.\left(\boldsymbol{h}_{1}, h_{2}\right) \in \zeta\right\}$ and $\zeta_{2, \boldsymbol{h}_{1}}=\left\{h_{2} \in \mathcal{F}_{2}:\left(\boldsymbol{h}_{1}, h_{2}\right) \in \zeta\right\} .|\zeta|$ denotes the cardinality of the set $\zeta$. We let $s_{(T)}$ (dependent on $T$ ) denote the number of nonzero entries in the true tensor coefficient, i.e., $s_{(T)}=\left\|\boldsymbol{\Gamma}_{(T)}^{(0)}\right\|_{0}$. Let $e_{\max }(\cdot)$ and $e_{\min }(\cdot)$ denote the largest and smallest eigenvalues of a square matrix, respectively.

Since the shrinkage prior on $\boldsymbol{\Gamma}_{(T)}$ assigns zero probability at the point zero, the exact number of nonzero elements of $\boldsymbol{\Gamma}_{(T)}$ is always $m_{(T)} \prod_{j=1}^{D} p_{j,(T)}$. A meaningful comparison with the value $s_{(T)}$ is made by considering $\tilde{s}_{(T)}$, the number of elements of $\boldsymbol{\Gamma}_{(T)}$ exceeding in absolute value a threshold $a_{T}$, which will be specified later. In other words, only elements with absolute values larger than $a_{T}$ will be treated as significant and counted towards non-zero entries.

Define $\mathcal{B}_{T}=\left\{\right.$ At least $\tilde{s}_{(T)}$ absolute values of $\boldsymbol{\Gamma}_{(T)}$ are greater than $\left.a_{T}\right\}$, $\mathcal{C}_{T}=\left\{\boldsymbol{\Gamma}_{(T)}:\left\|\boldsymbol{\Gamma}_{(T)}-\boldsymbol{\Gamma}_{(T)}^{(0)}\right\|_{2}>\epsilon\right\}$ and $\mathcal{A}_{T}=\mathcal{B}_{T} \cup \mathcal{C}_{T}$. Further suppose $\pi_{T}(\cdot)$ and $\Pi_{T}(\cdot)$ are the prior and posterior densities of $\boldsymbol{\Gamma}_{(T)}$ with $T$ observations, so that

$$
\Pi_{T}\left(\mathcal{A}_{T}\right)=\frac{\int_{\mathcal{A}_{T}} f\left(\boldsymbol{Y}_{1}, \ldots, \boldsymbol{Y}_{T} \mid \boldsymbol{\Gamma}_{(T)}\right) \pi_{T}\left(\boldsymbol{\Gamma}_{(T)}\right)}{\int f\left(\boldsymbol{Y}_{1}, \ldots, \boldsymbol{Y}_{T} \mid \boldsymbol{\Gamma}_{(T)}\right) \pi_{T}\left(\boldsymbol{\Gamma}_{(T)}\right)},
$$

where $f\left(\boldsymbol{Y}_{1}, \ldots, \boldsymbol{Y}_{T} \mid \boldsymbol{\Gamma}_{(T)}\right)$ is the joint density of $\boldsymbol{Y}_{1}, \ldots, \boldsymbol{Y}_{T}$ under the proposed model. This article intends to show

$$
\Pi_{T}\left(\mathcal{A}_{T}\right) \rightarrow 0 \text {, a.s., when } T \rightarrow \infty \text {. }
$$

\subsection{Main results}

The following theorem shows that (1.1) holds under mild sufficient conditions on $s_{(T)}, \tilde{s}_{(T)}$ and $p_{j,(T)} \mathrm{s}$. The proof of the theorem is given in the appendix. 
Theorem 1.1. Denote $p_{(T)}=m_{(T)} \prod_{j=1}^{D} p_{j,(T)}$. Let

(a) $\boldsymbol{\Gamma}_{k,(T)}^{(0)}$ assumes a rank- $R_{0}$ PARAFAC decomposition, $\boldsymbol{\Gamma}_{k,(T)}^{(0)}=\sum_{r=1}^{R_{0}} \boldsymbol{\gamma}_{1, k,(T)}^{0(r)} \circ$ $\cdots \circ \gamma_{D, k,(T)}^{0(r)}$, for $k=1, . ., m_{(T)}$, with $R>R_{0}$ and $\left\|\gamma_{j, k,(T)}^{0(r)}\right\|<\infty ;$

(b) $\left\|\boldsymbol{\Gamma}_{k,(T)}^{(0)}\right\|_{0}=s_{(T)}$, with $s_{(T)} \log \left(p_{(T)}\right)=o(T)$;

(c) $\tilde{s}_{(T)} \log \left(p_{(T)}\right)=o(T)$;

(d) $m_{(T)} \sum_{j=1}^{D} p_{j,(T)} \log \left(p_{j,(T)}\right)=o(T)$;

(e) There exists $\lambda_{0}, \lambda_{1}>0$ s.t. $e_{\min }\left(\boldsymbol{X}_{\nabla}^{\prime} \boldsymbol{R}^{-1} \boldsymbol{X}_{\nabla}\right) \geq T \lambda_{0}^{2}$ and $e_{\max }\left(\boldsymbol{X}_{\nabla}^{\prime} \boldsymbol{R}^{-1} \boldsymbol{X}_{\nabla}\right) \leq$ $T \lambda_{1}^{2}$, for any set $\nabla \subseteq\left\{1, \ldots, m_{(T)}\right\}$, where $\boldsymbol{X}_{\nabla}$ is a submatrix of $\boldsymbol{X}=\left[\boldsymbol{x}_{1}^{\prime}\right.$ : $\left.\cdots: \boldsymbol{x}_{T}^{\prime}\right]^{\prime}$ with columns corresponding to the indices $\nabla$.

Under conditions (a)-(e), (1.1) holds with $a_{T}=\frac{\epsilon}{2 p_{(T)}}$.

Remark: Condition (a) in Theorem 1.1 assumes a low-rank decomposition for the true tensor coefficient. This is a mild condition as most applications allow low-rank structure for the true tensor coefficients. Regarding condition (b), note that $s_{(T)}$ is the sparsity of the true tensor and $p_{(T)}$ is the total number of cells in the tensor. When the tensor is just a scalar, i.e., the tensor regression reduces to an ordinary high dimensional regression with $m_{(T)}$ predictors, the condition reduces to $s_{(T)} \log \left(m_{(T)}\right)=o(T)$, which is a typical assumption in ordinary high dimensional regression, see Song and Liang (2017). Condition (c) also assumes the same condition for the "near sparsity" in the estimated $\boldsymbol{\Gamma}_{(T)}$ in the sense of $\mathcal{B}_{T}$. Condition (d) in Theorem 1.1 requires that $m_{(T)} \sum_{j=1}^{D} p_{j,(T)}$ grows sublinearly with sample size $T$. However, the number of cells $m_{(T)} \prod_{j=1}^{D} p_{j,(T)}$ in the tensor $\boldsymbol{\Gamma}_{(T)}$ can grow at a rate much faster than the sample size $T$. Hence, the modeling framework allows large tensor responses even for moderate sample sizes. Condition (e) is equivalent to a lower bounded compatibility number condition assumed in the theoretical study of ordinary high dimensional regression, see Song and Liang (2017); Castillo et al. (2015). Finally, condition (e) also ensures that $e_{\max }\left(\boldsymbol{X}^{\prime} \boldsymbol{R}^{-1} \boldsymbol{X}\right)$ grows sub-linearly with $T$.

\section{Proofs of theoretical results}

The proof of Theorem 1.1 relies in part on the existence of exponentially consistent sequence of tests.

Definition An exponentially consistent sequence of test functions $\Phi_{T}$ for testing $H_{0}: \boldsymbol{\Gamma}_{(T)}=\boldsymbol{\Gamma}_{(T)}^{(0)}$ vs. $H_{1}: \boldsymbol{\Gamma}_{(T)} \in \mathcal{A}_{T}$ satisfies

$$
E_{\boldsymbol{\Gamma}_{(T)}^{(0)}}\left(\Phi_{T}\right) \leq c_{1} \exp \left(-b_{1} T\right), \quad \sup _{\boldsymbol{\Gamma}_{(T)} \in \mathcal{A}_{T}} E_{\boldsymbol{\Gamma}_{(T)}}\left(1-\Phi_{T}\right) \leq c_{2} \exp \left(-b_{2} T\right)
$$

for some $c_{1}, c_{2}, b_{1}, b_{2}>0$.

Theorem 2.1. There exist an exponentially consistent sequence of tests $\Phi_{T}$ for testing $H_{0}: \boldsymbol{\Gamma}_{(T)}=\boldsymbol{\Gamma}_{(T)}^{(0)}$ vs. $H_{1}: \boldsymbol{\Gamma}_{(T)} \in \mathcal{A}_{T}$. 
Proof. Let $\zeta \in \mathcal{F}_{1} \times \mathcal{F}_{2}$. For any $\boldsymbol{h}_{1} \in \zeta_{1}$, let $\hat{\boldsymbol{\Gamma}}_{(T), \boldsymbol{h}_{1}, \zeta_{2, h_{1}}}=\left(\boldsymbol{X}_{\zeta_{2, h_{1}}}^{\prime} \boldsymbol{R}^{-1} \boldsymbol{X}_{\zeta_{2, h_{1}}}\right)^{-1} \boldsymbol{X}_{\zeta_{2, h_{1}}}^{\prime} \boldsymbol{R}^{-1} \boldsymbol{y}_{\boldsymbol{h}_{1}}$, where $\boldsymbol{y}_{\boldsymbol{h}_{1}}=\left(Y_{1, \boldsymbol{h}_{1}}, \ldots, Y_{T, \boldsymbol{h}_{1}}\right)^{\prime}$ and $\boldsymbol{X}_{\zeta_{2, h_{1}}}$ is a $T \times\left|\zeta_{2, \boldsymbol{h}_{1}}\right|$ dimensional matrix whose $t$ th row is given by $\left(\boldsymbol{x}_{j, t}: j \in \zeta_{2, \boldsymbol{h}_{1}}\right)$. Define a test function $\Phi_{T}=$ $\max _{|\zeta| \leq \tilde{s}_{T}+s_{T}, \zeta \supseteq \zeta^{(0)}} 1\left\{\left\|\hat{\boldsymbol{\Gamma}}_{(T), \zeta}-\boldsymbol{\Gamma}_{(T), \zeta}^{(0)}\right\|_{2}>\epsilon / 4\right\}$. In what follows, we will show that $\Phi_{T}$ is an exponentially consistent sequence of tests.

$$
\begin{aligned}
& E_{\boldsymbol{\Gamma}_{(T)}^{(0)}}\left(\Phi_{T}\right) \leq \sum_{|\zeta| \leq \tilde{s}_{(T)}+s_{(T)}, \zeta \supseteq \zeta^{(0)}} P\left(\left\|\hat{\boldsymbol{\Gamma}}_{(T), \zeta}-\boldsymbol{\Gamma}_{(T), \zeta}^{(0)}\right\|_{2}>\epsilon / 4\right) \\
& \leq \sum_{|\zeta| \leq \tilde{s}_{(T)}+s_{(T)}, \zeta \supseteq \zeta^{(0)}} P\left(\sum_{\boldsymbol{h} \in \zeta_{1}}\left(\hat{\boldsymbol{\Gamma}}_{(T), \boldsymbol{h}, \zeta_{2, \boldsymbol{h}}}-\boldsymbol{\Gamma}_{(T), \boldsymbol{h}, \zeta_{2, \boldsymbol{h}}}^{(0)}\right)^{\prime}\left(\hat{\boldsymbol{\Gamma}}_{(T), \boldsymbol{h}, \zeta_{2, \boldsymbol{h}}}-\boldsymbol{\Gamma}_{(T), \boldsymbol{h}, \zeta_{2, \boldsymbol{h}}}^{(0)}\right)>\epsilon^{2} / 16\right) \\
& \leq \sum_{|\zeta| \leq \tilde{s}_{(T)}+s_{(T)}, \zeta \supseteq \zeta^{(0)}} P\left(\sum_{\boldsymbol{h} \in \zeta_{1}}\left(\hat{\boldsymbol{\Gamma}}_{(T), \boldsymbol{h}, \zeta_{2, \boldsymbol{h}}}-\boldsymbol{\Gamma}_{(T), \boldsymbol{h}, \zeta_{2, \boldsymbol{h}}}^{(0)}\right)^{\prime}\left(\boldsymbol{X}_{\zeta_{2, \boldsymbol{h}}}^{\prime} \boldsymbol{R}^{-1} \boldsymbol{X}_{\zeta_{2, \boldsymbol{h}}}\right)\left(\hat{\boldsymbol{\Gamma}}_{(T), \boldsymbol{h}, \zeta_{2, \boldsymbol{h}}}-\boldsymbol{\Gamma}_{(T), \boldsymbol{h}, \zeta_{2, \boldsymbol{h}}}^{(0)}\right)>\right. \\
& =\sum_{|\zeta| \leq \tilde{s}_{(T)}+s_{(T)}, \zeta \supseteq \zeta^{(0)}} P\left(\sum_{0}^{2} \epsilon^{2} / 16\right) \\
& =\sum_{|\zeta| \leq \tilde{s}_{(T)}+s_{(T)}, \zeta \supseteq \zeta^{(0)}} P\left(\chi_{\left|\zeta_{2, \boldsymbol{h}}\right|}^{2}>T \lambda_{0}^{2} \epsilon^{2} / 16\right) \\
& \left.\sum_{|\zeta|}^{2}>T \lambda_{0}^{2} \epsilon^{2} / 16\right) \leq\left(\begin{array}{c}
p_{(T)} \\
\tilde{s}_{(T)}+s_{(T)}
\end{array}\right) \exp \left(-T \lambda_{0}^{2} \epsilon^{2} / 16\right),
\end{aligned}
$$

where the last inequality follows from Lemma A.1 and A.2 in Song and Liang

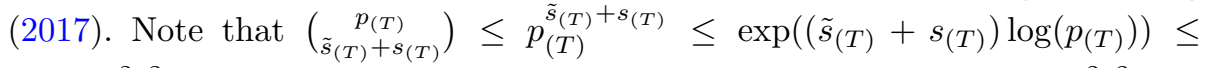
$\exp \left(T \lambda_{0}^{2} \epsilon^{2} / 32\right)$, by assumptions (b) and (c). Thus $E_{\boldsymbol{\Gamma}_{(T)}^{(0)}}\left(\Phi_{T}\right) \leq \exp \left(-T \lambda_{0}^{2} \epsilon^{2} / 32\right)$.

Let $\tilde{\zeta}=\zeta^{(0)} \cup\left\{\left(\boldsymbol{h}_{1}, h_{2}\right):\left|\Gamma_{(T), \boldsymbol{h}_{1}, h_{2}}\right| \geq a_{T}\right\}$

$$
\begin{aligned}
& \sup _{\boldsymbol{\Gamma}_{(T)} \in \mathcal{A}_{T}} E_{\boldsymbol{\Gamma}_{(T)}}\left(1-\Phi_{T}\right) \leq \sup _{\boldsymbol{\Gamma}_{(T)} \in \mathcal{A}_{T}} E_{\boldsymbol{\Gamma}_{(T)}}\left(1-1\left\{\left\|\hat{\boldsymbol{\Gamma}}_{(T), \tilde{\zeta}}-\boldsymbol{\Gamma}_{(T), \tilde{\zeta}}^{(0)}\right\|_{2}>\epsilon / 4\right\}\right) \\
& =\sup _{\boldsymbol{\Gamma}_{(T)} \in \mathcal{A}_{T}} P_{\boldsymbol{\Gamma}_{(T)}}\left(\left\|\hat{\boldsymbol{\Gamma}}_{(T), \tilde{\zeta}}-\boldsymbol{\Gamma}_{(T), \tilde{\zeta}}^{(0)}\right\|_{2} \leq \epsilon / 4\right) .
\end{aligned}
$$

Under $\mathcal{A}_{T},\left\|\boldsymbol{\Gamma}_{(T), \tilde{\zeta}}-\boldsymbol{\Gamma}_{(T), \tilde{\zeta}}^{(0)}\right\|_{2} \geq\left\|\boldsymbol{\Gamma}_{(T)}-\boldsymbol{\Gamma}_{(T)}^{(0)}\right\|_{2}-\left\|\boldsymbol{\Gamma}_{(T), \tilde{\zeta}^{c}}-\boldsymbol{\Gamma}_{(T), \tilde{\zeta}^{c}}^{(0)}\right\|_{2} \geq \epsilon-$ $a_{T} p_{T} \geq \epsilon / 2$. Where the last inequality follows due to the fact $\boldsymbol{\Gamma}_{(T), \tilde{\zeta}^{c}}^{(0)}=\mathbf{0}$ and $\left|\Gamma_{(T), \boldsymbol{h}_{1}, h_{2}}\right| \leq a_{T}$ for $\left(\boldsymbol{h}_{1}, h_{2}\right) \in \tilde{\zeta}^{c}$. 
Using the above fact

$$
\begin{aligned}
& \sup _{\boldsymbol{\Gamma}_{(T)} \in \mathcal{A}_{T}} E_{\boldsymbol{\Gamma}_{(T)}}\left(1-\Phi_{T}\right) \leq \sup _{\boldsymbol{\Gamma}_{(T)} \in \mathcal{A}_{T}} P_{\boldsymbol{\Gamma}_{(T)}}\left(\left\|\hat{\boldsymbol{\Gamma}}_{(T), \tilde{\zeta}}-\boldsymbol{\Gamma}_{(T), \tilde{\zeta}}^{(0)}\right\|_{2} \leq \epsilon / 4\right) \\
& \leq \sup _{\boldsymbol{\Gamma}_{(T)} \in \mathcal{A}_{T}} P_{\boldsymbol{\Gamma}_{(T)}}\left(\left\|\hat{\boldsymbol{\Gamma}}_{(T), \tilde{\zeta}}-\boldsymbol{\Gamma}_{(T), \tilde{\zeta}}\right\|_{2} \geq-\left\|\hat{\boldsymbol{\Gamma}}_{(T), \tilde{\zeta}}-\boldsymbol{\Gamma}_{(T), \tilde{\zeta}}^{(0)}\right\|_{2}+\left\|\boldsymbol{\Gamma}_{(T), \tilde{\zeta}}-\boldsymbol{\Gamma}_{(T), \tilde{\zeta}}^{(0)}\right\|_{2}\right) \\
& \leq \sup _{\boldsymbol{\Gamma}_{(T)} \in \mathcal{A}_{T}} P_{\boldsymbol{\Gamma}_{(T)}}\left(\left\|\hat{\boldsymbol{\Gamma}}_{(T), \tilde{\zeta}}-\boldsymbol{\Gamma}_{(T), \tilde{\zeta}}\right\|_{2} \geq \epsilon / 4\right) \\
& \leq \sup _{\boldsymbol{\Gamma}_{(T)} \in \mathcal{A}_{T}} P\left(\sum_{\boldsymbol{h} \in \zeta_{1}}\left(\hat{\boldsymbol{\Gamma}}_{(T), \boldsymbol{h}, \zeta_{2, \boldsymbol{h}}}-\boldsymbol{\Gamma}_{(T), \boldsymbol{h}, \zeta_{2, \boldsymbol{h}}}\right)^{\prime}\left(\boldsymbol{X}_{\zeta_{2, \boldsymbol{h}}}^{\prime} \boldsymbol{R}^{-1} \boldsymbol{X}_{\zeta_{2, \boldsymbol{h}}}\right)\left(\hat{\boldsymbol{\Gamma}}_{(T), \boldsymbol{h}, \zeta_{2, \boldsymbol{h}}}-\boldsymbol{\Gamma}_{(T), \boldsymbol{h}, \zeta_{2, \boldsymbol{h}}}\right)>T \lambda_{0}^{2} \epsilon^{2} / 16\right) \\
& \leq \sup _{\boldsymbol{\Gamma}_{(T)} \in \mathcal{A}_{T}} P\left(\sum_{\boldsymbol{h} \in \zeta_{1}} \chi_{\left|\zeta_{2, \boldsymbol{h}}\right|}^{2}>T \lambda_{0}^{2} \epsilon^{2} / 16\right) \\
& \leq P\left(\chi_{|\zeta|}^{2}>T \lambda_{0}^{2} \epsilon^{2} / 16\right) \leq \exp \left(-T \lambda_{0}^{2} \epsilon^{2} / 16\right) .
\end{aligned}
$$

Hence $\Phi_{T}$ is a exponentially consistent sequence of tests.

Next, we provide a bound on the discrepancy between the true and fitted tensor.

Theorem 2.2. Let $\mathcal{K}(\theta)=-\log \left\{\Pi_{T}\left(\boldsymbol{\Gamma}_{(T)}:\left\|\boldsymbol{\Gamma}_{(T)}-\boldsymbol{\Gamma}_{(T)}^{(0)}\right\|_{\infty}<\theta\right)\right\}$ and $\tilde{\boldsymbol{\gamma}}_{j, k, v_{j},(T)}=$ $\left(\gamma_{j, k, v_{j},(T)}^{(1)}, \ldots, \gamma_{j, k, v_{j},(T)}^{(R)}\right)^{\prime}$, and $\tilde{\boldsymbol{\gamma}}_{j, k, v_{j},(T)}^{(0)}=\left(\gamma_{j, k, v_{j},(T)}^{0(1)}, \ldots, \gamma_{j, k, v_{j},(T)}^{0(R)}\right)^{\prime}, \gamma_{j, k, v_{j},(T)}^{0(r)}=$ 0 for $r \in\left\{R_{0}+1, \ldots, R\right\}, R>R_{0}$. For $k=1, \ldots, m_{(T)}$, assume that $\Delta_{\boldsymbol{v}, k}$ is a positive root of the equations given, for all $\boldsymbol{v} \in \mathcal{F}_{1} \times \mathcal{F}_{2}$, by

$$
\begin{aligned}
& x\left(x+\left\|\tilde{\gamma}_{2, k, v_{2},(T)}^{(0)}\right\|\right) \cdots\left(x+\left\|\tilde{\gamma}_{D, k, v_{D},(T)}^{(0)}\right\|\right)+\left\|\tilde{\gamma}_{1, k, v_{1},(T)}^{(0)}\right\| x\left(x+\left\|\tilde{\boldsymbol{\gamma}}_{2, k, v_{2},(T)}^{(0)}\right\|\right) \cdots\left(x+\left\|\tilde{\gamma}_{D, k, v_{D},(T)}^{(0)}\right\|\right) \\
& +\cdots+x\left\|\tilde{\gamma}_{2, k, v_{2},(T)}^{(0)}\right\| \cdots\left\|\tilde{\gamma}_{D, k, v_{D},(T)}^{(0)}\right\|-\theta=0,
\end{aligned}
$$

and $\Delta=\min _{\boldsymbol{v}, k} \Delta_{\boldsymbol{v}, k}$. Then, for some constant $C$,

$$
\begin{aligned}
\mathcal{K}(\theta) & \leq\left(R m_{(T)} \sum_{j=1}^{D} p_{j,(T)}\right) \ln \left\{(2 \pi R)^{1 / 2} /(2 \Delta)\right\}-\ln (C)+R m_{(T)} \sum_{j=1}^{D} \ln \left\{\Gamma\left(a_{\lambda}\right) / \Gamma\left(a_{\lambda}+p_{j,(T)}\right)\right\} \\
& +\sum_{k=1}^{m_{(T)}} \sum_{j=1}^{D} \sum_{r=1}^{R_{0}}\left(a_{\lambda}+p_{j,(T)}\right) \ln \left[b_{\lambda}+\sum_{v_{j}=1}^{p_{j,(T)}}\left\{\left(\gamma_{j, k, v_{j},(T)}^{0(r)}\right)^{2}+2 \Delta^{2}\right\}^{1 / 2}\right] \\
& +\left(R-R_{0}\right) m_{(T)} \sum_{j=1}^{D}\left(a_{\lambda}+p_{j,(T)}\right) \ln \left(b_{\lambda}+p_{j,(T)} 2^{1 / 2} \Delta\right) .
\end{aligned}
$$


Proof.

$$
\begin{aligned}
& \left|\Gamma_{\boldsymbol{v}, k,(T)}-\Gamma_{\boldsymbol{v}, k,(T)}^{(0)}\right|=\left|\sum_{r=1}^{R} \gamma_{1, k, v_{1},(T)}^{(r)} \cdots \gamma_{D, k, v_{D},(T)}^{(r)}-\sum_{r=1}^{R} \gamma_{1, k, v_{1},(T)}^{0(r)} \cdots \gamma_{D, k, v_{D},(T)}^{0(r)}\right| \\
= & \left|\sum_{r=1}^{R}\left\{\left(\gamma_{1, k, v_{1},(T)}^{(r)}-\gamma_{1, k, v_{1},(T)}^{0(r)}\right) \prod_{j \neq 1} \gamma_{j, k, v_{j},(T)}^{(r)}+\cdots+\left(\gamma_{D, k, v_{D},(T)}^{(r)}-\gamma_{D, k, v_{D},(T)}^{0(r)}\right) \prod_{j \neq D} \gamma_{j, k, v_{j},(T)}^{0(r)}\right\}\right| \\
\leq & \left\|\tilde{\boldsymbol{\gamma}}_{1, k, v_{1},(T)}-\tilde{\boldsymbol{\gamma}}_{1, k, v_{1},(T)}^{(0)}\right\|_{2} \prod_{j \neq 1}\left\|\tilde{\boldsymbol{\gamma}}_{j, k, v_{j},(T)}\right\|_{2}+\cdots+\left\|\tilde{\boldsymbol{\gamma}}_{D, k, v_{D},(T)}-\tilde{\boldsymbol{\gamma}}_{D, k, v_{D},(T)}^{(0)}\right\|_{2} \prod_{j \neq D}\left\|\tilde{\boldsymbol{\gamma}}_{j, k, v_{j},(T)}^{(0)}\right\|_{2},
\end{aligned}
$$

Note that (2.1) can be written as $\mathrm{g}_{\boldsymbol{v}, k}(x)=0$, where

$$
\mathrm{g}_{\boldsymbol{v}, k}(x)=a_{D, k, \boldsymbol{v}} x^{D}+\cdots+a_{1, k, \boldsymbol{v}} x-a_{0, k, \boldsymbol{v}}
$$

and the $a_{j, k, \boldsymbol{v}}$ 's are suitably chosen to match the coefficient of $x^{j}$ in (2.1). By Cauchy's bound on the roots of polynomials, Eq. (2.1) has only one positive root, namely the real $\Delta_{\boldsymbol{v}, k}$ that satisfies $\Delta_{\boldsymbol{v}, k} \leq 1+\max _{j=0, \ldots, D}\left|a_{j, k, \boldsymbol{v}}\right|$, for all $\boldsymbol{v}$ and $k$. From (2.1), the fact that $\left\|\tilde{\boldsymbol{\gamma}}_{j, k, v_{j},(T)}-\tilde{\boldsymbol{\gamma}}_{j, k, v_{j},(T)}^{(0)}\right\|<\Delta$ for all $v_{j} \in\left\{1, \ldots, p_{j,(T)}\right\}, j \in\{1, \ldots, D\}$ and $k \in\left\{1, \ldots, m_{(T)}\right\}$ implies

$$
\left|\Gamma_{\boldsymbol{v}, k,(T)}-\Gamma_{\boldsymbol{v}, k,(T)}^{(0)}\right| \leq \mathrm{g}_{\boldsymbol{v}, k}(\Delta)+\theta \leq \mathrm{g}_{\boldsymbol{v}, k}\left(\Delta_{\boldsymbol{v}, k}\right)+\theta=\theta,
$$

which leads to $\left\|\boldsymbol{\Gamma}_{(T)}-\boldsymbol{\Gamma}_{(T)}^{(0)}\right\|_{\infty}<\theta$. Hence

$\Pi_{T}\left(\boldsymbol{\Gamma}_{(T)}:\left\|\boldsymbol{\Gamma}_{(T)}-\boldsymbol{\Gamma}_{(T)}^{(0)}\right\|_{\infty}<\theta\right) \geq \Pi_{T}\left(\forall_{k \in\left\{1, \ldots, m_{(T)}\right\}} \forall_{j \in\{1, \ldots, D\}} \forall_{v_{j} \in\left\{1, \ldots, p_{j,(T)}\right\}}\left\|\tilde{\boldsymbol{\gamma}}_{j, k, v_{j},(T)}-\tilde{\boldsymbol{\gamma}}_{j, k, v_{j},(T)}^{(0)}\right\|_{2}<\Delta\right)$.

We will bound the right-hand side from below.

$\Pi_{T}\left(\forall_{k \in\left\{1, \ldots, m_{(T)}\right\}} \forall_{j \in\{1, \ldots, D\}} \forall_{v_{j} \in\left\{1, \ldots, p_{j,(T)}\right\}}\left\|\tilde{\gamma}_{j, v_{j}, T}-\tilde{\gamma}_{j, v_{j}, T}^{(0)}\right\|_{2}<\Delta \mid \forall_{k \in\left\{1, \ldots, m_{(T)}\right\}}\left\{\phi_{r, k}\right\}, \tau_{k},\left\{W_{j r, k}\right\}\right)$

$$
\begin{aligned}
& =\prod_{k=1}^{m_{(T)}} \prod_{j=1}^{D} \prod_{v_{j}=1}^{p_{j,(T)}}\left[\exp \left\{-\sum_{r=1}^{R}\left(\gamma_{j, k, v_{j},(T)}^{0(r)}\right)^{2} /\left(2 w_{j r, k, v_{j}} \phi_{r, k} \tau_{k}\right)\right\} \Pi_{T}\left(\left\|\tilde{\gamma}_{j, k, v_{j},(T)}\right\|<\Delta / 2 \mid\left\{\phi_{r, k}\right\}, \tau_{k},\left\{W_{j r, k}\right\}\right)\right] \\
& \geq \prod_{k=1}^{m_{(T)}} \prod_{j=1}^{D} \prod_{v_{j}=1}^{p_{j,(T)}}\left[\operatorname { e x p } \{ - \sum _ { r = 1 } ^ { R } ( \gamma _ { j , k , v _ { j } , ( T ) } ^ { 0 ( r ) } ) ^ { 2 } / ( 2 w _ { j r , k , v _ { j } } \phi _ { r , k } \tau _ { k } ) \} \prod _ { r = 1 } ^ { R } \left[\exp \left\{-\Delta^{2} /\left(\phi_{r, k} \tau_{k} w_{j r, k, v_{j}}\right)\right\}\right.\right. \\
& \left.\geq \prod_{k=1}^{m_{(T)}} \prod_{j=1}^{D} \prod_{v_{j}=1}^{p_{j,(T)}} \prod_{r=1}^{R}\left[(2 \Delta) /\left(2 \pi R \phi_{r, k} \tau_{k} w_{j r, k, v_{j}}\right)^{1 / 2}\right]\right]
\end{aligned}
$$

where Step 2 follows from Anderson's lemma. Integrating out the $w_{j r, k, v_{j}}$ 's, we obtain

$$
\begin{aligned}
& \Pi\left(\forall_{k \in\left\{1, \ldots, m_{(T)}\right\}} \forall_{j \in\{1, \ldots, D\}} \forall_{v_{j} \in\left\{1, \ldots, p_{j,(T)}\right\}}\left\|\tilde{\gamma}_{j, k, v_{j},(T)}-\tilde{\gamma}_{j, k, v_{j},(T)}^{(0)}\right\|<\Delta \mid \tau_{k},\left\{\phi_{r, k}\right\},\left\{\lambda_{j r, k}\right\}\right) \\
\geq & \prod_{k=1}^{m_{(T)}} \prod_{r=1}^{R} \prod_{j=1}^{D}\left[\left\{\left(2 \Delta \lambda_{j r, k}\right) /\left(R \phi_{r, k} \tau_{k}\right)^{1 / 2}\right\}^{p_{j,(T)}} \exp \left[-\lambda_{j r, k} \sum_{v_{j}=1}^{p_{j,(T)}}\left\{\left(\gamma_{j, k, v_{j},(T)}^{0(r)}\right)^{2}+2 \Delta^{2}\right\}^{1 / 2} /\left(\phi_{r, k} \tau_{k}\right)^{1 / 2}\right]\right] .
\end{aligned}
$$


Integrating out the $\lambda_{j r, k}$ 's, we then get

$$
\begin{aligned}
& \Pi_{T}\left(\forall_{k \in\left\{1, \ldots, m_{(T)}\right\}} \forall_{j \in\{1, \ldots, D\}} \forall_{v_{j} \in\left\{1, \ldots, p_{j,(T)}\right\}}\left\|\tilde{\gamma}_{j, k, v_{j},(T)}-\tilde{\gamma}_{j, k, v_{j},(T)}^{(0)}\right\|<\Delta \mid \tau_{k},\left\{\phi_{r, k}\right\}\right) \\
& \geq \prod_{k=1}^{m_{(T)}} \prod_{r=1}^{R} \prod_{j=1}^{D}\left[\left\{(2 \Delta) /\left(R \phi_{r, k} \tau_{k}\right)^{1 / 2}\right\}^{p_{j,(T)}} \frac{\Gamma\left(a_{\lambda}+p_{j,(T)}\right)}{\left.\left[b_{\lambda}+\sum_{v_{j}=1}^{p_{j,(T)}}\left\{\left(\gamma_{j, k, v_{j},(T)}^{0(r)}\right)^{2}+2 \Delta^{2}\right\}^{1 / 2}\left(\phi_{r, k} \tau_{k}\right)^{-1 / 2}\right]^{a_{\lambda}+p_{j,(T)}}\right]}\right] \\
& \quad\left\{b_{\lambda}^{\left.a_{\lambda} / \Gamma\left(a_{\lambda}\right)\right\}^{R(D)}}\right] \\
& \geq \prod_{k=1}^{m_{(T)}} \prod_{r=1}^{R} \prod_{j=1}^{D}\left[\left\{(2 \Delta) /\left(R \phi_{r, k} \tau_{k}\right)^{1 / 2}\right\}^{p_{j,(T)}}\left\{b_{\lambda}^{a_{\lambda}} / \Gamma\left(a_{\lambda}\right)\right\} \frac{\Gamma\left(a_{\lambda}+p_{j,(T)}\right)\left(\phi_{r, k} \tau_{k}\right)^{\left(a_{\lambda}+p_{j,(T)}\right) / 2} \mathbf{1}\left\{\tau_{k} \in(0,1)\right\}}{\left[b_{\lambda}+\sum_{v_{j}=1}^{\left.p_{j,(T)}\left\{\left(\gamma_{j, k, v_{j},(T)}^{0(r)}\right)^{2}+2 \Delta^{2}\right\}^{1 / 2}\right]^{a_{\lambda}+p_{j,(T)}}}\right]}\right.
\end{aligned}
$$

Integrating our $\phi_{r, k}$ 's together we obtain,

$$
\begin{aligned}
& \Pi_{T}\left(\forall_{k \in\left\{1, \ldots, m_{(T)}\right\}} \forall_{j \in\{1, \ldots, D\}} \forall_{v_{j} \in\left\{1, \ldots, p_{j,(T)}\right\}}\left\|\tilde{\gamma}_{j, k, v_{j},(T)}-\tilde{\gamma}_{j, k, v_{j},(T)}^{(0)}\right\|<\Delta \mid \tau_{k}\right) \\
& \geq \prod_{k=1}^{m_{(T)}} \prod_{r=1}^{R} \prod_{j=1}^{D}\left[\left\{(2 \Delta) /\left(R \tau_{k}\right)^{1 / 2}\right\}^{p_{j,(T)}}\left\{b_{\lambda}^{a_{\lambda}} / \Gamma\left(a_{\lambda}\right)\right\} \frac{\Gamma\left(a_{\lambda}+p_{j,(T)}\right) \tau_{k}^{\left(a_{\lambda}+p_{j,(T)}\right) / 2} \mathbf{1}\left\{\tau_{k} \in(0,1)\right\}}{\left[b_{\lambda}+\sum_{i_{j}=1}^{p_{j,(T)}}\left\{\left(\gamma_{j, k, v_{j},(T)}^{0(r)}\right)^{2}+2 \Delta^{2}\right\}^{1 / 2}\right]^{a_{\lambda}+p_{j,(T)}}}\right] \\
& \prod_{r=1}^{R-1}\left[\frac{\operatorname{Beta}\left(D, \alpha_{k}+D(R-r)\right)}{\operatorname{Beta}\left(1, \alpha_{k}\right)}\right],
\end{aligned}
$$

where $\operatorname{Beta}\left(m_{1}, m_{2}\right)$ is the integrating constant for the Beta density with parameters $m_{1}$ and $m_{2}$. Finally, integrating out $\tau_{k}$, leads to

$$
\begin{aligned}
& \Pi_{T}\left(\forall_{k \in\left\{1, \ldots, m_{(T)}\right\}} \forall_{j \in\{1, \ldots, D\}} \forall_{v_{j} \in\left\{1, \ldots, p_{j,(T)}\right\}}\left\|\tilde{\gamma}_{j, k, v_{j},(T)}-\tilde{\gamma}_{j, k, v_{j},(T)}^{(0)}\right\|<\Delta\right) \\
& \geq \prod_{k=1}^{m_{(T)}} \prod_{j=1}^{D}\left\{\Gamma\left(a_{\lambda}+p_{j,(T)}\right) / \Gamma\left(a_{\lambda}\right)\right\}^{R} \prod_{k=1}^{m_{(T)}} \prod_{j=1}^{D} \prod_{r=1}^{R}\left[b_{\lambda}+\sum_{v_{j}=1}^{p_{j,(T)}}\left\{\left(\gamma_{j, k, v_{j},(T)}^{0(r)}\right)^{2}+2 \Delta^{2}\right\}^{1 / 2}\right]^{-a_{\lambda}-p_{j,(T)}} \\
& \left\{2 \Delta /(2 \pi R)^{1 / 2}\right\}^{R m_{(T)} \sum_{j=1}^{D} p_{j,(T)} C^{-1}}
\end{aligned}
$$

for some constant $C$. Hence

$$
\begin{aligned}
\mathcal{K}(\theta) & \leq-\log \left[\Pi_{T}\left(\forall_{k \in\left\{1, \ldots, m_{(T)}\right\}} \forall_{j \in\{1, \ldots, D\}} \forall_{v_{j} \in\left\{1, \ldots, p_{j,(T)}\right\}}\left\|\tilde{\gamma}_{j, k, v_{j},(T)}-\tilde{\gamma}_{j, k, v_{j},(T)}^{(0)}\right\|<\Delta\right)\right] \\
& \leq\left(R m_{(T)} \sum_{j=1}^{D} p_{j,(T)}\right) \ln \left\{(2 \pi R)^{1 / 2} /(2 \Delta)\right\}-\ln (C)+R m_{(T)} \sum_{j=1}^{D} \ln \left\{\Gamma\left(a_{\lambda}\right) / \Gamma\left(a_{\lambda}+p_{j,(T)}\right)\right\} \\
& +\sum_{k=1}^{m_{(T)}} \sum_{j=1}^{D} \sum_{r=1}^{R_{0}}\left(a_{\lambda}+p_{j,(T)}\right) \ln \left[b_{\lambda}+\sum_{v_{j}=1}^{p_{j,(T)}}\left\{\left(\gamma_{j, k, v_{j},(T)}^{0(r)}\right)^{2}+2 \Delta^{2}\right\}^{1 / 2}\right] \\
& +\left(R-R_{0}\right) m_{(T)} \sum_{j=1}^{D}\left(a_{\lambda}+p_{j,(T)}\right) \ln \left(b_{\lambda}+p_{j,(T)} 2^{1 / 2} \Delta\right) .
\end{aligned}
$$


Under assumptions (a)-(f), the R.H.S is $o(T)$. Thus, we present the next theorem whose proof follows immediately from Theorem 2.2 .

Theorem 2.3. For any constant $\theta>0$, under conditions $(a)-(f)$ of Theorem 1.1, $\mathcal{K}(\theta)=o(T)$.

\section{Proof of Theorem 1.1}

Proof.

$$
\begin{aligned}
\Pi_{T}\left(\mathcal{A}_{T}\right) & =\frac{\int_{\mathcal{A}_{T}} f\left(\boldsymbol{Y}_{1}, . ., \boldsymbol{Y}_{T} \mid \boldsymbol{\Gamma}_{(T)}\right) \pi_{T}\left(\boldsymbol{\Gamma}_{(T)}\right)}{\int f\left(\boldsymbol{Y}_{1}, . ., \boldsymbol{Y}_{T} \mid \boldsymbol{\Gamma}_{(T)}\right) \pi_{T}\left(\boldsymbol{\Gamma}_{(T)}\right)}=\frac{\int_{\mathcal{A}_{T}} \frac{f\left(\boldsymbol{Y}_{1}, . ., \boldsymbol{Y}_{T} \mid \boldsymbol{\Gamma}_{(T)}\right)}{f\left(\boldsymbol{Y}_{1}, . ., \boldsymbol{Y}_{T} \mid \boldsymbol{\Gamma}_{(T)}^{(0)}\right)} \pi_{T}\left(\boldsymbol{\Gamma}_{(T)}\right)}{\int \frac{f\left(\boldsymbol{Y}_{1}, ., \boldsymbol{Y}_{T} \mid \boldsymbol{\Gamma}_{(T)}\right)}{f\left(\boldsymbol{Y}_{1, \ldots,}, \boldsymbol{Y}_{T} \mid \boldsymbol{\Gamma}_{(T)}^{(0)}\right)} \pi_{T}\left(\boldsymbol{\Gamma}_{(T)}\right)} \\
& =\frac{\mathcal{N}_{T}}{\mathcal{D}_{T}} \leq \Phi_{T}+\left(1-\Phi_{T}\right) \frac{\mathcal{N}_{T}}{\mathcal{D}_{T}}
\end{aligned}
$$

where $\Phi_{T}$ is the exponentially consistent sequence of tests given by Lemma 2.1. Note that

$P_{\boldsymbol{\Gamma}_{(T)}^{(0)}}\left(\Phi_{T}>\exp \left(-T \lambda_{0}^{2} \epsilon^{2} / 64\right)\right) \leq E_{\boldsymbol{\Gamma}_{(T)}^{(0)}}\left(\Phi_{T}\right) \exp \left(T \lambda_{0}^{2} \epsilon^{2} / 64\right) \leq \exp \left(-T \lambda_{0}^{2} \epsilon^{2} / 64\right)$.

Therefore $\sum_{T=1}^{\infty} P_{\boldsymbol{\Gamma}_{(T)}^{(0)}}\left(\Phi_{T}>\exp \left(-T \lambda_{0}^{2} \epsilon^{2} / 64\right)\right)<\infty$. Applying Borel-Cantelli lemma $P_{\boldsymbol{\Gamma}_{(T)}^{(0)}}\left(\Phi_{T}>\exp \left(-T \lambda_{0}^{2} \epsilon^{2} / 64\right)\right.$ infinitely often $)=0$. Thus,

$$
\Phi_{T} \rightarrow 0 \quad \text { a.s. }
$$

In addition, we have

$$
\begin{aligned}
& E_{\boldsymbol{\Gamma}_{(T)}^{(0)}}\left(\left(1-\Phi_{T}\right) \mathcal{N}_{T}\right)=\int\left(1-\Phi_{T}\right) \int_{\mathcal{A}_{T}} \frac{f\left(\boldsymbol{Y}_{1}, . ., \boldsymbol{Y}_{T} \mid \boldsymbol{\Gamma}_{(T)}\right)}{f\left(\boldsymbol{Y}_{1}, . ., \boldsymbol{Y}_{T} \mid \boldsymbol{\Gamma}_{(T)}^{(0)}\right)} \pi_{T}\left(\boldsymbol{\Gamma}_{(T)}\right) f\left(\boldsymbol{Y}_{1}, . ., \boldsymbol{Y}_{T} \mid \boldsymbol{\Gamma}_{(T)}^{(0)}\right) \\
& =\int_{\mathcal{A}_{T}} \int\left(1-\Phi_{T}\right) f\left(\boldsymbol{Y}_{1}, . ., \boldsymbol{Y}_{T} \mid \boldsymbol{\Gamma}_{(T)}\right) \pi_{T}\left(\boldsymbol{\Gamma}_{(T)}\right) \leq \sup _{\boldsymbol{\Gamma}_{(T)} \in \mathcal{A}_{T}} E_{\boldsymbol{\Gamma}_{(T)}}\left(1-\Phi_{T}\right) \leq \exp \left(-T \lambda_{0}^{2} \epsilon^{2} / 16\right) .
\end{aligned}
$$

Applying Borel-Cantelli lemma, $P_{\boldsymbol{\Gamma}_{(T)}^{(0)}}\left(\left(1-\Phi_{T}\right) \mathcal{N}_{T} \exp \left(T \lambda_{0}^{2} \epsilon^{2} / 32\right)>\exp \left(-T \lambda_{0}^{2} \epsilon^{2} / 64\right)\right.$ infinitely often $)=$ 0 so

$$
\exp \left(T \lambda_{0}^{2} \epsilon^{2} / 32\right)\left(1-\Phi_{T}\right) \mathcal{N}_{T} \rightarrow 0 \quad \text { a.s.. }
$$

Note that $\mathcal{D}_{T}=\int \frac{f\left(\boldsymbol{Y}_{1}, ., \boldsymbol{Y}_{T} \mid \boldsymbol{\Gamma}_{(T)}\right)}{f\left(\boldsymbol{Y}_{1}, . ., \boldsymbol{Y}_{T} \mid \boldsymbol{\Gamma}_{(T)}^{(0)}\right)} \pi_{T}\left(\boldsymbol{\Gamma}_{(T)}\right)$. Let $\tilde{b}=\lambda_{0}^{2} \epsilon^{2} / 32$. Consider the set

$$
\begin{aligned}
& \mathcal{H}_{T}=\left\{\boldsymbol{\Gamma}_{(T)}: \frac{1}{T} \log \left[\frac{f\left(\boldsymbol{Y}_{1}, . ., \boldsymbol{Y}_{T} \mid \boldsymbol{\Gamma}_{(T)}^{(0)}\right)}{f\left(\boldsymbol{Y}_{1}, . ., \boldsymbol{Y}_{T} \mid \boldsymbol{\Gamma}_{(T)}\right)}\right]<v\right\}, \text { for } v=\tilde{b} / 2 . \\
& \exp (\tilde{b} T) \mathcal{D}_{T} \geq \exp (\tilde{b} T) \int_{\mathcal{H}_{T}} \exp \left(-T \frac{1}{T} \log \frac{f\left(\boldsymbol{Y}_{1}, . ., \boldsymbol{Y}_{T} \mid \boldsymbol{\Gamma}_{(T)}^{(0)}\right)}{f\left(\boldsymbol{Y}_{1}, . ., \boldsymbol{Y}_{T} \mid \boldsymbol{\Gamma}_{(T)}\right)}\right) \pi_{T}\left(\boldsymbol{\Gamma}_{(T)}\right) \geq \exp ((\tilde{b}-\tilde{b} / 2) T) \Pi_{T}\left(\mathcal{H}_{T}\right) .
\end{aligned}
$$


In view of $(2.2),(2.3)$ and $(2.4)$, it is enough to show that $-\log \left(\Pi_{T}\left(\mathcal{H}_{T}\right)\right) \leq$ $T \tilde{b} / 8$.

$$
\begin{aligned}
& \frac{1}{T} \log \left[\frac{f\left(\boldsymbol{Y}_{1}, . ., \boldsymbol{Y}_{T} \mid \boldsymbol{\Gamma}_{(T)}^{(0)}\right)}{f\left(\boldsymbol{Y}_{1}, . ., \boldsymbol{Y}_{T} \mid \boldsymbol{\Gamma}_{(T)}\right)}\right]=\frac{1}{T}\left[-\frac{1}{2} \sum_{\boldsymbol{v}}\left(\boldsymbol{y}_{\boldsymbol{v}}-\sum_{k=1}^{m_{(T)}} \boldsymbol{\Gamma}_{\boldsymbol{v}, k,(T)}^{(0)} \boldsymbol{x}_{k}\right)^{\prime} \boldsymbol{R}^{-1}\left(\boldsymbol{y}_{\boldsymbol{v}}-\sum_{k=1}^{m_{(T)}} \boldsymbol{\Gamma}_{\boldsymbol{v}, k,(T)}^{(0)} \boldsymbol{x}_{k}\right)\right. \\
& \left.+\frac{1}{2} \sum_{\boldsymbol{v}}\left(\boldsymbol{y}_{\boldsymbol{v}}-\sum_{k=1}^{m_{(T)}} \boldsymbol{\Gamma}_{\boldsymbol{v}, k,(T)} \boldsymbol{x}_{k}\right)^{\prime} \boldsymbol{R}^{-1}\left(\boldsymbol{y}_{\boldsymbol{v}}-\sum_{k=1}^{m_{(T)}} \boldsymbol{\Gamma}_{\boldsymbol{v}, k,(T)} \boldsymbol{x}_{k}\right)\right] . \\
& \Pi_{T}\left(\boldsymbol{\Gamma}_{(T)}: \frac{1}{T} \log \left[\frac{f\left(\boldsymbol{Y}_{1}, . ., \boldsymbol{Y}_{T} \mid \boldsymbol{\Gamma}_{(T)}^{(0)}\right)}{f\left(\boldsymbol{Y}_{1}, . ., \boldsymbol{Y}_{T} \mid \boldsymbol{\Gamma}_{(T)}\right)}\right]<v\right) \\
& \geq \Pi_{T}\left(\boldsymbol{\Gamma}_{(T)}:\left|\frac{1}{2 T} \sum_{\boldsymbol{v}} \sum_{k=1}^{m_{(T)}}\left(\boldsymbol{\Gamma}_{\boldsymbol{v}, k,(T)}-\boldsymbol{\Gamma}_{\boldsymbol{v}, k,(T)}^{(0)}\right)^{\prime} \boldsymbol{x}_{k}^{\prime} \boldsymbol{R}^{-1} \boldsymbol{x}_{k}\left(\boldsymbol{\Gamma}_{\boldsymbol{v}, k,(T)}-\boldsymbol{\Gamma}_{\boldsymbol{v}, k,(T)}^{(0)}\right)\right|<v\right) \\
& \geq \Pi_{T}\left(\boldsymbol{\Gamma}_{(T)}:\left\|\boldsymbol{\Gamma}_{(T)}-\Gamma_{(T)}^{(0)}\right\|_{2}^{2}<2 v / \lambda_{1}^{2}\right) \\
& \geq \Pi_{T}\left(\boldsymbol{\Gamma}_{(T)}:\left\|\boldsymbol{\Gamma}_{(T)}-\boldsymbol{\Gamma}_{(T)}^{(0)}\right\|_{\infty}<\sqrt{2 v / \lambda_{1}^{2}}\right) \geq \exp (-T \tilde{b} / 8),
\end{aligned}
$$

where the third line follows from assumption (e) of Theorem 3.1 and last inequality is immediate by applying Theorem 2.3.

\section{Posterior Sampling Algorithm}

This posterior sampling algorithm can be done efficiently by sampling the regionspecific variables in parallel.

(1) Draw $\alpha$ via a Griddy-Gibbs algorithm as follows:

(a) For each possible value of $\alpha$, draw a sample of size $\mathcal{M}$ from the posterior distributions of $\phi_{r}$ and $\tau$.

(b) Evaluate the prior density using each of these individual samples using the previous iteration values for all other parameters.

(c) Average these densities together for each possible value for $\alpha$ in the grid, and then sample one value using the averaged densities as weights.

(2) Using the posterior full conditional kernel of

$$
\begin{aligned}
p\left(\xi_{r} \mid \boldsymbol{\gamma}_{\cdot, r}, \mathbf{W}_{\cdot, r}, \xi_{-r}, \tau\right) \propto & \xi_{r}^{-\sum p_{j} / 2}\left(1-\xi_{r}\right)^{-(R-r) \sum p_{j} / 2} \\
& \times \exp \left\{-\frac{1}{\tau}\left[\frac{1}{\xi_{r}} \sum_{j=1}^{D}\left(\boldsymbol{\gamma}_{j, r}^{T} \mathbf{W}_{j, r}^{-1} \boldsymbol{\gamma}_{j, r}\right)\right.\right. \\
& \left.\left.+\sum_{k=r+1}^{R} \frac{1}{\xi_{k} \prod_{\ell=r}^{k-1}\left(1-\xi_{\ell}\right)} \sum_{j=1}^{D}\left(\boldsymbol{\gamma}_{j, r}^{T} \mathbf{W}_{j, r}^{-1} \boldsymbol{\gamma}_{j, r}\right)\right]\right\},
\end{aligned}
$$


draw $\xi_{r}^{(s)}$ for sample $s$ using a Metropolis-Hastings step with a normal proposal distribution with mean $\xi_{r}^{(s-1)}$ and variance $0.01^{2}$. The value for the variance was chosen such that the datasets tested showed decent mixing. After drawing $\xi_{1}, \ldots, \xi_{R-1}$, set $\phi_{r}=\xi_{r} \times \prod_{k=1}^{r-1}\left(1-\xi_{k}\right)$, and set $\phi_{R}=$ $1-\sum_{r=1}^{R-1} \phi_{r}$.

(3) Draw each $\tau$ from a generalized inverse Gaussian distribution, $g I G(\nu, \chi, \psi)$, where

$$
\nu=a_{\tau}-\frac{R \sum p_{j}}{2}, \quad \chi=\sum_{r=1}^{R} \frac{1}{\phi_{r}}\left(\sum_{j=1}^{D} \boldsymbol{\gamma}_{j}^{(r)^{\prime}} \mathbf{W}_{j}^{(r)-1} \boldsymbol{\gamma}_{j}^{(r)}\right), \quad \psi=2 b_{\tau}
$$

(4) Draw each $\lambda_{j}^{(r)}$ from a

$$
\operatorname{Gamma}\left(a_{\lambda}+p_{j}, b_{\lambda}+\frac{1}{\sqrt{\phi_{r} \tau}} \sum_{\ell=1}^{p_{j}}\left|\beta_{j, \ell}^{(r)}\right|\right)
$$

(5) Draw each $w_{j, \ell}^{(r)}$ from a generalized Inverse Gaussian distribution, $g I G(\nu, \chi, \psi)$, where

$$
\nu=\frac{1}{2} \quad \chi=\frac{\gamma_{j, \ell}^{(r) 2}}{\tau \phi_{r}} \quad \psi=\lambda_{j}^{(r) 2}
$$

(6) When $D=2$, draw each $\gamma_{j, z}^{(r)}$ from a normal distribution with variance

$$
\boldsymbol{\Lambda}=\left(\frac{1}{\phi_{r} \tau w_{j, z}^{(r)}}+\frac{n \sum x_{t}^{2} \gamma_{-j}^{(r)^{\prime}} \gamma_{-j}^{(r)}}{\sigma_{y}^{2}}\right)^{-1}
$$

and mean

$$
\boldsymbol{\mu}=\boldsymbol{\Lambda} \frac{\sum x_{t} \boldsymbol{\gamma}_{-j}^{(r)^{\prime}} \hat{\mathbf{y}}_{t}}{\sigma_{y}^{2}}
$$

where

$$
\hat{\mathbf{y}}_{t}=\mathbf{y}_{t}-x_{t} \sum_{\ell \neq r} \gamma_{1}^{(\ell)} \circ \gamma_{2}^{(\ell)}
$$

(7) Draw $\kappa$ from a truncated normal distribution with a lower bound -1 , an upper bound of 1 , a variance of

$$
\frac{\sigma_{\epsilon}^{2}}{\sum_{t=2}^{T} \sum_{\ell} \epsilon_{t-1, \ell}^{2}}
$$

and mean of

$$
\frac{\sum_{t=2}^{T} \sum_{\ell} \epsilon_{t, \ell} \epsilon_{t-1, \ell}}{\sum t=2^{T} \sum_{\ell} \epsilon_{t-1, \ell}^{2}}
$$


where

$\epsilon_{t, \ell}=y_{t, l}-\Gamma_{\ell} x_{t}$.

(8) Draw $\sigma_{\epsilon}^{2}$ from an inverse gamma distribution with shape parameter

$$
a_{\epsilon}+\frac{\prod p_{j}}{2},
$$

and scale parameter

$$
b_{\epsilon}+\frac{1}{2} \sum_{t=2}^{T} \sum_{\ell}\left(\epsilon_{t, \ell}-\kappa \epsilon_{t-1, \ell}\right)^{2}
$$

Following this algorithm, the MCMC converges rapidly to the region of the maximum likelihood estimator.

\section{Monteral Neurological Institute Atlas}

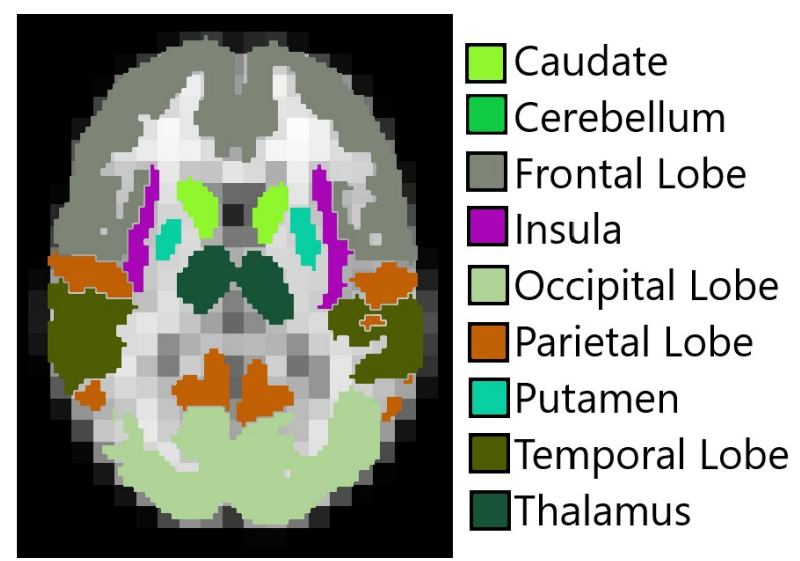

FIG 1. An illustration of the atlas used in the real data analysis for the axial slices used in the low- and high-resolution analysis figures.

\section{References}

Castillo, I., Schmidt-Hieber, J., Van der Vaart, A., et al. (2015). "Bayesian linear regression with sparse priors." The Annals of Statistics, 43(5): 1986-2018. 3

Song, Q. and Liang, F. (2017). "Nearly optimal Bayesian shrinkage for high dimensional regression." arXiv preprint arXiv:1712.08964. 3, 4

Van der Vaart, A. W. and Van Zanten, H. (2009). "Adaptive Bayesian estimation using a Gaussian random field with inverse gamma bandwidth." The Annals of Statistics, 37(5B): 2655-2675. 1

- (2011). "Information rates of nonparametric Gaussian process methods." Journal of Machine Learning Research, 12(Jun): 2095-2119. 2 